\title{
Risk behaviour and STD acquisition in genitourinary clinic attenders who have travelled
}

\author{
S Hawkes, G J Hart, E Bletsoe, C Shergold, A M Johnson
}

\begin{abstract}
Objective-To investigate the travel history of clients presenting at a genitourinary medicine (GUM) clinic in order to assess the contribution made by sexual partnerships abroad to STD transmission in the UK.
\end{abstract}

Subjects-386 old and new clients who attended during a 3-month period and who had travelled abroad in the 3 months preceding their visit.

Methods-All participating clients selfcompleted a confidential questionnaire, the results of which were then linked to their clinical diagnosis (if any).

Results-25\% of participants reported a new sexual partner during their most recent trip abroad. In comparison to those not reporting a new partner, they were more likely to be male, travelling alone, to have visited the clinic previously and to have no regular sexual partner. Two-thirds reported never or inconsistently using condoms with these new partners. A total of $11.6 \%$ of the STDs diagnosed in the study participants may have been acquired abroad.

Conclusion-We have found a high rate of new sexual relationships reported by attendees at our GUM clinic, and a low rate of reported condom use. With high HIV incidence rates in many tourist regions, the need for further studies to establish the true extent of imported STDs in the UK is a priority, and primary prevention campaigns to inform travellers are of paramount importance.

(Genitourin Med 1995;71:351-354)

Keywords: travel; risk; STD acquisition

\section{Introduction}

The relationship between sexual behaviour, sexually acquired infection and travel has long been noted in both popular culture and vernacular. Until recently, however, the strength of this relationship had not been quantified empirically. In the mid-1970s the existence of penicillinase producing $N$ gonorrhoeae (PPNG) was first recognised, and epidemiologists showed that the majority of infections reported in Europe had been acquired outside the borders of the continent. ${ }^{1-3}$ Although this situation has now changed and an increasing proportion of PPNG infections are acquired in the local community, ${ }^{4}$ it is not clear what proportion of other sexually transmitted infections are acquired abroad.

Despite recognition of the role of travel in the spread of sexually transmitted infections, and its potential for influencing sexual behaviour, very few studies have investigated the risks of acquiring a sexually transmitted infection associated with travel. ${ }^{5}$ In the United Kingdom only two previous studies have been published which detail the rate of risky behaviour during travel. In Nottingham Gillies et al conducted an anonymous postal survey among a sample of 1030 adults aged 16-40 years. ${ }^{6}$ These adults were randomly selected from the register of a general medical practice. With a response rate of $56 \%$ the authors found that two thirds of participants had undertaken foreign travel in the preceding year. Five per cent reported sexual intercourse with a new partner whilst abroad, and these people were more likely to be male, single, young, and travelling without a partner. We have previously reported the results of a survey carried out among returning travellers at the Hospital for Tropical Diseases, London. ${ }^{7}$ Eighteen per cent of participants had a new sexual partner or partners during their most recent trip abroad and $5.7 \%$ of these contracted a sexually transmitted disease during their most recent travels, emphasising the lack of safer sexual practices in this group of people.

Epidemiological surveillance data are available which indicate the percentage of HIV infections in the United Kingdom which have been acquired abroad. Since $198475 \%$ of heterosexually acquired HIV infections reported in the United Kingdom have been in people who either lived in or visited WHO Pattern II countries where heterosexual transmission of the virus is common. ${ }^{8}$ These data include people who originate from outside the UK and British people with heterosexual exposure abroad. ${ }^{9}$ As the HIV pandemic spreads an understanding of sexual mixing patterns between British residents and those resident abroad is fundamental to an understanding of potential HIV transmission. Yet there are few studies available to indicate which people put themselves at risk of exposure to HIV and other sexually transmitted infections.

We report here what we believe to be the first study to investigate the travel history of those presenting at a GUM clinic in an attempt to assess the contribution of sexual partnerships abroad to STD transmission. 
Table 1 Sexual orientation of respondents and rates of new partners acquisition

\begin{tabular}{llll}
\hline & $\begin{array}{l}\text { New partner in } \\
3 \text { months pre-trip }\end{array}$ & $\begin{array}{l}\text { New partner } \\
\text { during trip }\end{array}$ & $\begin{array}{l}\text { New partner } \\
\text { post-trip }\end{array}$ \\
\hline $\begin{array}{l}\text { Homosexual men } \\
(\mathrm{n}=101)\end{array}$ & $75 \%(74 / 99)$ & $44 \%(44 / 100)$ & $53 \%(53 / 100)$ \\
$\begin{array}{l}\text { Heterosexual men } \\
(\mathrm{n}=74)\end{array}$ & $42 \%(31 / 73)$ & $25 \%(18 / 71)$ & $19 \%(14 / 73)$ \\
$\begin{array}{l}\text { Women } \\
(\mathrm{n}=199)\end{array}$ & $27 \%(52 / 193)$ & $18 \%(35 / 195)$ & $11 \%(22 / 193)$ \\
\hline
\end{tabular}

\section{Methods}

Study parameters From March to June 1993 all patients seen at the outpatients department of a central London GUM clinic (James Pringle House) who were eligible for syphilis serology (that is, all new patients and all old patients with a new condition) were asked if they had travelled out of the United Kingdom in the preceding three months. If the patient had recently travelled then s/he was asked to selfcomplete a questionnaire relating to sexual behaviour in the three months pre-travel, during travel and since their return to the UK. The questionnaires were confidential but not anonymous and subsequent diagnostic data were linked to the behavioural questionnaire. The diagnosis codes used are the KC60 returns-the nationally accepted coding system for collecting information on the numbers and types of diagnoses made in GUM clinics. ${ }^{10}$

Statistical Analysis The chi square test was used to test for evidence of an association between the categorical variables; Fisher's exact test was used where the expected frequencies were small. Odds ratios (OR) and their corresponding $95 \%$ confidence intervals (CI) were produced to present the "risk" factors for the acquisition of new sexual partners abroad.

The study had the approval of the Middlesex Hospital Clinical Investigations Committee, and participation in the study was entirely voluntary.

\section{Results}

During the three months of the study, 3978 patients attended James Pringle House and were given a diagnosis code from the KC60 reference list. ${ }^{10}$ In the same time period, 2556 patients had blood taken for syphilis serology. Of these, 462 (18\%) were eligible for participation in the study as they had travelled abroad within the last three months. Overall we obtained 386 completed questionnaires during the study period, giving a response rate of $83 \%$. Three hundred and twenty questionnaires had a clinic identifying number on them to which we were able to link a diagnosis code.

The majority of respondents were female (207; 54\%), and the mean age of all respon- dents was $30 \cdot 2$ years $(S D=8 \cdot 4)$. The majority of those having blood taken for syphilis serology were male (1375; 54\%), but their overall mean age was similar to those completing questionnaires. Two hundred and seventyseven respondents $(72 \%)$ were single, but $71 \%(274 / 386)$ had a current regular sex partner, although only $8 \%(30 / 386)$ were married and $10 \%(38 / 386)$ were co-habiting. Of the 179 male respondents, $57 \%(101 / 176)$ had had sexual intercourse, with another man at some time, and $25 \%(44 / 178)$ had never had sexual intercourse with a woman; all of these had had sex with a man. Three per cent of female respondents $(6 / 200)$ had had sex with another woman, and 5 of these 6 women had also had sex with a man.

The most commonly cited reason for travel was holiday $(239 / 385 ; 62 \%)$, followed by travel for business $(113 / 385 ; 29 \%$ ) (respondents were able to list more than one reason for travel). More than a third had travelled alone $(113 / 308 ; 37 \%)$ during their most recent trip. Of those travelling with someone else, $28 \%(85 / 308)$ were with a sexual partner, $19 \%$ (58/308) were in a group, $17 \%(53 / 308)$ with a friend and $6 \%(13 / 213)$ with a colleague. Sixty-eight per cent (263/386) of the respondents were born in the United Kingdom, 23\% came from Western Europe, North America or Australia/New Zealand. Only $9 \%$ of participants originated from outside these areas.

One quarter of respondents $(98 / 386 ; 25 \%)$ reported having one or more new sexual partners during their most recent trip abroad; this compares with $42 \%(162 / 386)$ with a new partner(s) in the 3 months pre-travel and $23 \%$ $(89 / 386)$ with a new sexual partner(s) since returning home. The sexual orientation of the respondents and proportions with new partners are shown in table 1 . It can be seen that homosexual men were much more likely to report new partners in all intervals, and heterosexual men were more likely to report new partners than heterosexual women.

Table 2 shows that the 98 people who reported having a new sexual partner during their most recent trip abroad were more likely to be male, to be travelling alone, to report a new sexual partner since returning to the UK, to have visited the clinic previously and to be less likely to have a current regular sexual partner when compared with the 279 not reporting new travel abroad. On other parameters there was no significant difference between the two groups of people.

The number of new sexual partners reported overall by the respondents was 542 in the three months pre-travel, 275 during travel and 176 since returning. We asked detailed information on a maximum of 5 new partners at each tem-

Table 2 Characteristics of those reporting new partners compared with those not reporting new partners

\begin{tabular}{llll}
\hline & 98 with new partners & 279 without new partners & p value OR (CI) \\
\hline Male & $64 \%(63 / 98)$ & $40 \%(111 / 279)$ & $<0 \cdot 0001$ OR $=2 \cdot 72(1 \cdot 65-4 \cdot 52)$ \\
Travelling alone & $62 \%(45 / 73)$ & $30 \%(68 / 229)$ & $<0 \cdot 0001$ OR $=3 \cdot 81(2 \cdot 12-6 \cdot 85)$ \\
Has a current regular sexual partner & $46 \%(44 / 96)$ & $80 \%(222 / 279)$ & $<0 \cdot 0001$ OR $=0 \cdot 22(0 \cdot 13-0 \cdot 37)$ \\
New sexual partmer since returning & $42 \%(41 / 98)$ & $17 \%(48 / 275)$ & $<0 \cdot 0001$ OR $=3 \cdot 4(1 \cdot 98-5 \cdot 84)$ \\
Visiting for a sexual health check-up & $65 \%(63 / 98)$ & $46 \%(129 / 279)$ & $0 \cdot 0017$ OR $=2 \cdot 19(1 \cdot 32-3 \cdot 63)$ \\
Has visited clinic previously & $61 \%(60 / 98)$ & $42 \%(117 / 279)$ & 0.0015 OR $=2 \cdot 19(1 \cdot 33-3 \cdot 63)$ \\
\hline
\end{tabular}


Table 3 Comparison of travellers with an STD diagnosis and those with no STD detected

\begin{tabular}{llll}
\hline & 61 with an STD & 254 no STD & p value \\
\hline Male & $52 \%(32 / 61)$ & $44 \%(111 / 254)$ & NS \\
Homosexual/bisexual & $37 \%(22 / 59)$ & $25 \%(62 / 246)$ & $\mathrm{p}=0.08$ \\
No current sexual partner & $33 \%(20 / 61)$ & $28 \%(71 / 251)$ & $\mathrm{NS}$ \\
Travelled alone & $30 \%(14 / 47)$ & $37 \%(76 / 208)$ & $\mathrm{NS}$ \\
Attending because symptomatic & $78 \%(47 / 60)$ & $41 \%(103 / 252)$ & $\mathrm{p}<0.0001$ \\
First visit to clinic & $58 \%(35 / 60)$ & $52 \%(131 / 253)$ & $\mathrm{NS}$ \\
\hline
\end{tabular}

NS = Not significant.

poral point and thus have information relating to 364,203 and 161 partners respectively. Eighty-five participants who originated from WHO Pattern I countries (Western Europe, North America and Australasia, that is countries where the original spread of HIV infection was mainly found in the homosexual and injecting drug-using communities) had 156 new sexual partners abroad. Whilst the majority $(81 \% ; 127 / 156)$ of these new partners were also from WHO Pattern I countries, $18.6 \%$ $(29 / 156)$ were from outside these areas including $7 \%(11 / 156)$ from Asia and 1.3\% (2/156) from Sub-Saharan Africa.

The rate of payment for sex with a new partner was $1.6 \%(6 / 364)$ in the three months pre-travel, $5.4 \%(11 / 203)$ during travel and $0.6 \%(1 / 161)$ since returning. Of the five people who paid for sex with 11 new partners abroad, all 5 were men, and 4 were heterosexual ( 1 was bisexual). Thus $22 \%(4 / 18)$ of heterosexual men with new partners abroad paid for sex with those partners. The majority $(9 / 11 ; 81 \%)$ of their partners were from Asia, one was African and one was European. Two of the five men reported never or inconsistently using condoms with commercial sex workers. None of these men had a diagnosis of an STD on this clinic visit.

We asked respondents whether they used condoms during sexual activity with each new partner: over two thirds said that they used condoms occasionally or never with new partners. This figure was consistent for behaviour pre- $(68 \%)$, during $(69 \%)$ and post- $(76 \%)$ travel. The remaining $30 \%$ reported always using condoms with new partners.

In analysing the data relating to the diagnosis of sexually transmitted infections, we have looked at the results of the 386 who completed questionnaires: $315(82 \%)$ had a retrievable diagnosis code and thus had results available for analysis (table 3 ). Sixty-one people (19\%) had a diagnosis of a sexually transmitted infection, the rest had no abnormality detected. In comparing these 61 with the 254 without an infection, it was found that they were more

Table 4 Comparison of travellers and non-travellers with an STD diagnosis

\begin{tabular}{lcc}
\hline & Non-travellers & Travellers \\
STD Diagnosis & $n=2024(\%)$ & $n=315(\%)$ \\
\hline Any diagnosis & $465(23)$ & $61(19 \cdot 4)$ \\
Primary syphilis & $1(0 \cdot 05)$ & $0(0)$ \\
Gonorrhoea & $42(2 \cdot 1)$ & $5(1 \cdot 6)$ \\
Primary herpes simplex & $51(2 \cdot 5)$ & $4(1 \cdot 3)$ \\
Primary human papilloma virus & $100(4 \cdot 9)$ & $20(6 \cdot 4)$ \\
Chlamydia trachomatis & $69(3 \cdot 4)$ & $5(1 \cdot 6)$ \\
Non-specific urethritis & $141(7)$ & $19(6)$ \\
Trichomonas vaginalis & $9(0 \cdot 4)$ & $4(1 \cdot 3)$ \\
Non-specific epididymitis/PID & $24(1 \cdot 2)$ & $4(1 \cdot 3)$ \\
Asymptomatic HIV (1st presentation) & $27(1 \cdot 3)$ & $0(0)$ \\
Chancroid/LGV & $1(0 \cdot 05)$ & $0(0)$ \\
\hline
\end{tabular}

likely to be attending the clinic because they had symptoms rather than just for a sexual health check-up $(47 / 60$ v 103/252, $\mathrm{p}<0.0001$ ), and although they were more likely to be homosexual men this was a nonsignificant trend (22/59 $v 62 / 246, \mathrm{p}=0.08)$. Those with an STD diagnosis reported a slightly lower rate of condom use at all times (that is, pre, during and after trip with new partners) than those without a diagnosis, but the differences were not statistically significant.

Table 4 shows the percentage of travellers and non-travellers with an STD at their visit to the clinic. The STDs we have included in this analysis are only those which could have been acquired within the recent past. Thus, we have excluded recurrent problems and previously diagnosed infections from the data analysis. Overall, $11 \cdot 6 \%(61 / 526)$ of all STD primary infections occurred amongst those who had travelled abroad. This is the maximum proportion of infections attributable to infection abroad, and assumes that those with a new STD who had travelled abroad acquired that infection outside the UK.

\section{Discussion}

We found a high rate of sexual activity with new partners in people attending our STD clinic who have travelled. One quarter of the respondents had a new sexual partner during their most recent trip abroad. This figure is similar to that found in a study of the sexual risk behaviour of travellers reported from the Hospital for Tropical Diseases (HTD), London, ${ }^{7}$ but higher than that found in a survey of adults on a General Practice list (5\%). ${ }^{6}$ In the study of travellers at the HTD $18.6 \%$ of respondents had a new sexual partner(s) during their most recent trip abroad, and most of them did not use condoms consistently with their new partners. This is to be expected in a study of this kind since STD clinic attenders are likely to have placed themselves at recent risk and to have larger numbers of partners than the general population. ${ }^{11}$

Our results show a low reported consistent use of condoms: over two-thirds said they used condoms only occasionally or never with new partners, and this was the case for behaviour pre-, during and post-travel. This proportion may represent the worst case since a large number of our respondents were men who have sex with men and may be reporting oral sex without condoms. The group of men who reported homosexual activity $(n=101)$ reported the highest rates of sex with a new partner at the three temporal points. However, it is important to note that almost one quarter of heterosexual men and $18 \%$ of women reported a new sexual partner during their most recent trip abroad (again, a figure which corresponds to that found in our previous survey of travellers). As this was a self-completed questionnaire, we did not ask more detailed questions on specific types of sexual activity as this was found to have created a degree of confusion among some of the respondents in the pilot stage. Further research should investigate 
issues such as whether condom use is associated with countries visited, type of sexual behaviour and risk group.

As with all questionnaires relating to behaviour which may have occurred several months previously, there may be a certain level of recall bias within the reported results especially as we asked about sexual behaviour in the three months pre-travel. However, the effect of recall bias is likely to be systematic as people were asked about events within the same, recent time frame. Similarly, any bias would be expected to be similar for people of all types of sexual orientation, and would not account for differences in reported rates of sexual activity.

Whilst most people with new sexual partners abroad had partners from a similar geographical origin to themselves, almost one fifth of those from Pattern I countries had partners from outside this area. Five men paid for sex with their partners abroad, and the overall rate of payment for sex abroad among heterosexual men with new partners was $22 \%$-a figure which was higher than that reported either pre- or post-travel. Notably, 9/11 of the paid partners of these men were not from Pattern I countries. Rates of HIV infection among commercial sex workers in some areas outside WHO Pattern I countries range from $<2 \%$ to over $90 \%,,^{12}$ and with the reportedly low consistent use of condoms by our respondents some travellers may be putting themselves at high risk of HIV infection.

In general those people with a new sexual partner abroad were more likely to report higher rates of sexual activity since returning to the UK and to have made a previous visit to our clinic than those who did not have a new partner abroad (table 2). Like the Nottingham study, those with a new partner abroad were more likely to be single than those without a new partner. However, unlike the previously reported study we found no association with gender, reason for travel, whether travelling alone, or the age of the respondent. These figures illustrate that there is a group of attenders at our GUM clinic who may be at particular risk for acquiring a sexually transmitted infection abroad: a group with risky sexual behaviour in this country are likely to continue their pattern of activity when abroad, including in countries where the prevalence of some STDs (including HIV) may be much higher than in the UK.

Overall $19 \%(61 / 315)$ of our study participants had a diagnosis of a sexually transmitted infection. This figure did not differ significantly from that in the group of people who did not travel $(465 / 2024 ; 23 \%)$, nor did the rates of specific infections diagnosed show any significant differences, and there was no signifcant difference in the STD rates by respondents' country of origin. Up to $12 \%$ of new STDs in this clinic during the study period may have been as a result of new partnerships abroad. This is the maximum attributable figure and assumes that all persons with new STDs and a recent history of travel acquired their infections abroad. The 61 travellers with an STD were found to be different from those travellers without an STD: they were more likely to be homosexual men, and they were less likely to use condoms (although the latter finding was not statistically significantly different). The results are important in terms of health education as they indicate that homosexual men may still be taking risks with their sexual health and exposing themselves to the risk of STDs, including HIV, as well as potentially exposing individuals abroad to HIV.

Our study has highlighted the extent to which our respondents put themselves at risk of acquiring a sexually transmitted infection during their most recent trip abroad. Whilst the people visiting our clinic are not representative of the travelling public as a whole or even of all STD clinic attenders, it should be remembered that the number of visits made abroad by members of the British public is high. ${ }^{14}$ Thus even if the sexually active proportion of the total number of travellers is low, the absolute numbers of people at risk will be high. We have shown that in the study population those most at risk were people with a history of sexual behaviour which may previously have put them at risk of infection with an STD, and many had previously visited an STD clinic. This highlights the opportunity for health education about sexual health risks in any geographical location which can be incorporated into the visit of all attenders at a department of genitourinary medicine.

Furthermore, our results emphasise the need to repeat this type of survey in other clinics which may serve different types of GUM clinic attenders-for example, clinics with a lower percentage of homosexual men. It also suggests that improved surveillance mechanisms are required to assess the proportion of STDs which can be attributed to contact outside the UK. Only with a more detailed picture from different geographical areas in the UK can the true contribution of STDs acquired abroad be measured.

1 Philips I. Beta-lactamase producing, penicillin resistan gonococcus. Lancet 1976;2:656-7.

2 Reyn A. Drug susceptibility pattern of Neisseria gonorrhoeae. A worldwide review. Asian $\mathcal{F}$ Infect Dis 1977;1:1-14.

3 Lind I. Epidemiology of antibiotic resistant $N$ gonorrhoeae in industrialised and developing countries. Scand $\mathcal{F} \operatorname{Inf} D$ is 1990; Suppl.69:77-82.

4 The Public Health Laboratory Service Disease Surveillance and the Communicable Diseases (Scotland) Unit. Penicillinase-producing gonococci in Britain, 1983. BMF 1984;288: 1746 .

5 Hawkes SJ, Hart GJ. Travel, migration and HIV. AIDS Care 1993;5: 207-214.

6 Gillies P, Slack R, Stoddart N, Conway S. HIV-related risk behaviour in UK holiday-makers. AIDS 1992;6:339-42.

7 Hawkes S, Hart G J, Johnson A M, et al. D. Risk behaviour and HIV prevalence in international travellers. AIDS and HIV preval.

8 Communicable Disease Report. AIDS and HIV-1 infection in the United Kingdom: monthly report. Communicable Diseases Report 1995;5:13-16.

9 Noone A, Gill ON, Clark SE, Porter K. Travel, heterosexual intercourse and HIV-1 infection. Communicable Disease Review; 1991;1:R39-R43.

10 Department of Health New cases seen at NHS GUM clinics in England. Summary information from form KC60. 1992 Annual figures. London, Department of Health, 1992.

11 Johnson A M, Wadsworth J, Wellings K, Bradshaw S, Field J. Sexual lifestyle and HIV risk. Nature 1992;360:410-2.

12 Celentano DD, Akarasewi P, Sussman S. HIV-1 infection among lower class commercial sex workers in Chiang among lower class commercial sex
Mai, Thailand. AIDS 1994;8:533-8.

13 Nkowane BM. Prevalence and incidence of HIV infection in Africa: a review of data published in 1990. AIDS 1991; 5(suppl):S7-S15.

14 Central Statistical Office. Business Monitor: Overseas travel and tourism. HMSO 1989, MA6, London. 